\title{
Health disparities and equity in the era of COVID-19
}

www.cambridge.org/cts

\section{Implementation, Policy and Community Engagement Review Article}

Cite this article: Nana-Sinkam $\mathrm{P}$, Kraschnewski J, Sacco R, Chavez J, Fouad M, Gal T, AuYoung M, Namoos A, Winn R, Sheppard V, Corbie-Smith G, and BeharZusman V. Health disparities and equity in the era of COVID-19. Journal of Clinical and Translational Science 5: e99, 1-8. doi: 10.1017/ cts. 2021.23

Received: 5 January 2021

Revised: 28 February 2021

Accepted: 4 March 2021

\section{Keywords:}

Health disparities; health equity; COVID-19; clinical and translational research

\section{Address for correspondence:}

P. Nana-Sinkam, MD, Division of Pulmonary and Critical Care Medicine, Virginia

Commonwealth University, P.O. Box 980050 , Richmond, VA 23298-0050, USA.

Email: patrick.nana-sinkam@vcuhealth.org

(c) The Author(s), 2021. Published by Cambridge University Press on behalf of The Association for Clinical and Translational Science. This is an Open Access article, distributed under the terms of the Creative Commons AttributionNonCommercial-NoDerivatives licence (http:// creativecommons.org/licenses/by-nc-nd/4.0/), which permits non-commercial re-use, distribution, and reproduction in any medium, provided the original work is unaltered and is properly cited. The written permission of Cambridge University Press must be obtained for commercial re-use or in order to create a derivative work.

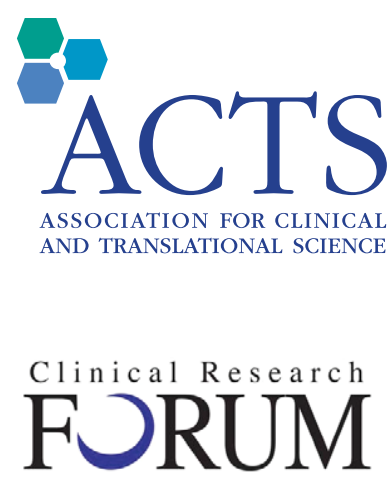

Analysis. Advocacy. Action.

\author{
Patrick Nana-Sinkam ${ }^{1}$, Jennifer Kraschnewski ${ }^{2}$, Ralph Sacco ${ }^{3}$, Jennifer Chavez ${ }^{4}$, \\ Mona Fouad ${ }^{5}$, Tamas Gal ${ }^{1}$ (1), Mona AuYoung ${ }^{6}$, Asmaa Namoos ${ }^{1}$, Robert Winn ${ }^{1}$, \\ Vanessa Sheppard ${ }^{1}$, Giselle Corbie-Smith ${ }^{7}$ and Victoria Behar-Zusman ${ }^{8}$
}

${ }^{1}$ Massey Cancer Center, Virginia Commonwealth University, Richmond, VA, USA; ${ }^{2}$ Division of General Internal Medicine, Penn State College of Medicine, Hershey, PA, USA; ${ }^{3}$ Department of Neurology, University of Miami Health, Miami, FL, USA; ${ }^{4}$ University of Miami School of Medicine, Miami, FL, USA; ${ }^{5}$ Division of Preventive Medicine, University of Alabama at Birmingham School of Medicine, Birmingham, AL, USA; ${ }^{6}$ Scripps Health, San Diego, CA, USA; ${ }^{7}$ Department of Social Medicine, University of North Carolina School of Medicine, Chapel Hill, NC, USA and ${ }^{8}$ School of Nursing and Health Studies, University of Miami, Coral Gables, FL, USA

\section{Abstract}

Over the last year, COVID-19 has emerged as a highly transmissible and lethal infection. As we address this global pandemic, its disproportionate impact on Black, Indigenous, and Latinx communities has served to further magnify the health inequities in access and treatment that persist in our communities. These sobering realities should serve as the impetus for reexamination of the root causes of inequities in our health system. An increased commitment to strategic partnerships between academic and nonacademic health systems, industry, local communities, and policy-makers may serve as the foundation. Here, we examine the impact of the recent COVID-19 pandemic on health care inequities and propose a strategic roadmap for integration of clinical and translational research into our understanding of health inequities.

\section{Underlying Causes of Health Inequities in the Era of COVID-19}

As of February 18, 2021, there were over 110 million reported cases of COVID-19 globally, with approximately 2.4 million deaths. There have been over 27 million reported cases and 490,000 deaths in the USA [1]. The convergence of this highly transmissible and deadly disease with ongoing social and economic inequities has placed a significant strain on our health care system. Simultaneously, disparities in risk of infection, morbidity, and mortality have magnified the inherent limitations to health care access and delivery. The disproportionate impact of the pandemic in Black, Indigenous, and Latinx communities has highlighted health inequities in access and treatment, social determinants of health, the need for the rapid and efficient development and delivery of testing and therapeutics, and the importance of implementation of best practices that reach our communities. Black and non-Hispanics Blacks account for 13\% of the US population, yet $17 \%$ of the deaths, while Hispanic/Latino account for $17 \%$ of the US population yet $22 \%$ of cases and $13.8 \%$ of deaths (Centers for Disease Control and Prevention (CDC), https:// covid.cdc.gov/covid-data-tracker/\#demographics). American Indian/Alaska Natives (AI/AN) currently account for $1.3 \%$ of cases, and $1 \%$ of deaths, and represent $2 \%$ of the US population. Among 340,059 cases with complete patient race/ethnicity data, the cumulative incidence among AI/AN persons in these 23 states was 594/100,000 AI/AN population compared with 169 per 100,000 White population. AI/AN persons with COVID-19 were younger (median age $=40$ years; interquartile range $[\mathrm{IQR}]=26-56$ years) than Whites (median age $=51$ years; $\mathrm{IQR}=32-67$ years) [2].The drivers of these sobering statistics are multifactorial. Structural racism laid the foundation for increased risk of COVID-19 in racial and ethnic minority communities. The impact of this racism has resulted in increased infectious spread among disenfranchised individuals due to long-standing limitations on the upward social and economic mobility of racial and ethnic minorities, disproportionate conscription to jobs that are considered essential that cannot be done from home, and limited protections for themselves and their families. Structural inequities have contributed to increased morbidity and mortality from coronavirus infection due to limited access to care, inadequacies in public policy, and a disproportionate burden of comorbidities carrying increased risk, including cardiovascular disease, diabetes, and lung diseases. These factors all intersect to define increased risk of both infection and resultant poor outcomes.

Historically, the roadmap for reducing health disparities has focused on interpersonal or behavioral factors with calls for increased cultural competency, decreased bias, and heightened awareness within the US health care system of the social and economic disadvantages faced by communities. Unfortunately, these efforts have not resulted in any significant reduction in 
disparities. The recent co-occurring events of the pandemic and wider recognition of structural racism have led to greater emphasis on the root causes of inequities. These efforts have included expansion of a diverse health care workforce, improved strategies for ensuring partnership between health care and public policy to improve access for those most marginalized, and increasing emphasis on disease prevention. A timely recommitment to clinical and translational research that focuses on disparities and health equity, for example, has included the use of comprehensive data elements from electronic medical records (EMRs) and data visualization to highlight COVID-19 disparities. This data visualization provides opportunity for better understanding trends in illness by race and ethnicity and thus informing new discoveries. In fact, the ongoing COVID-19 pandemic represents a unique opportunity for clinical and translational centers to strengthen partnerships with the community, policy decision-makers, and industry to reexamine and reaffirm the potential of translational research as a disrupter of health disparities. Here, we discuss the scope of health care inequities in the context of the COVID-19 pandemic, examine the current challenges in reducing gaps in health care disparities, and propose a strategic roadmap for the integration of new clinical models and translational research in the development of sustainable solutions to reducing disparities in health care.

\section{Recognizing and Addressing Challenges to Health Disparities in the Setting of COVID-19}

While distressing, the disproportionate impact of the pandemic on low-income and racial/ethnic minority communities is not surprising. There are well-established, long-standing disparities that negatively impact individuals from racial/ethnic, poor, or rural communities that are the product of a history of structural inequality. Formerly invisible to some, the people who stock our grocery shelves, deliver our mail, process our meat, clean our hospitals, drive our buses, care for our elderly, and harvest our vegetables are now labeled as "essential workers" [3]. Individuals from low-income individuals and racial/ethnic communities are overrepresented in the essential workforce and are more likely to live in multigenerational households [4] or densely clustered housing, and they are less likely to have access to sufficient health care or protective equipment, let alone sufficient sick leave or resources to quarantine safely. Even among essential workers, mortality from COVID-19 is higher among non-Hispanic/ Latinx Blacks compared to Whites [5]. Redlining and other discriminatory practices and policies [4] have created neighborhoods and communities that have limited health promoting opportunities, health care access, open spaces, clean air, good schools, and other resources to stay healthy. This is reflected in racial/ethnic minorities bearing some of the highest disease burden of comorbid diseases such as diabetes, cardiovascular diseases, stroke, asthma, HIV, cancer, liver, obesity, and kidney disease [6] - all risk factors that increase the risk for more severe cases of COVID-19 [7, 8]. More specifically, hypertension [9] and diabetes are noted as critical risk factors associated with increased COVID-19 hospitalizations and higher mortality rates. This means the very populations with the greatest risk of exposure (not by choice), with the greatest risk for illness (not by choice), also have the least access to protection (not by choice). To be clear, a confluence of structural factors - not individual level factors - has resulted in historically marginalized communities being the most impacted by COVID-19 in terms of health outcomes, economics, and social stigma.
Moreover, the COVID-19 pandemic has also exacerbated already disproportionate existing comorbidities. Fear and misinformation continue to delay ongoing care for chronic conditions, essential screenings for cancer and other conditions, and receipt of preventive services. Long term, these delays in routine care will undoubtedly lead to worsening morbidity and increase preventable deaths.

\section{Community Health Workers}

In the midst of the pandemic, it has become clear the important role that community health workers (CHWs) from underserved communities have played in the public health workforce. The American Public Health Association defines a $\mathrm{CHW}$ as a "frontline public health worker who is a trusted member of, and/or has an unusually close understanding of, the community served" [10]. The ability of CHWs to address health inequities has been clear and their role in addressing COVID-19 has become even more evident. However, to fully realize the potential of $\mathrm{CHWs}$ as a bridge from public health initiatives to communities, workforce development is needed to support the current and emerging challenges related to the pandemic $[11,12]$. CHWs have experienced a lack of resources and technology to support and refer clients with limited access to testing and hindered dissemination of services because of social distancing. Specifically, CHWs have experienced challenges with telehealth and videoconferencing technology and require resources to provide their services virtually to reach potential clients [13].

\section{Diagnostic Testing and Safety in the Era of COVID-19}

Although COVID-19 testing was recognized early on as critical for containing the virus, there was limited access to testing and testing resources due to delayed approvals from the Food and Drug Administration (FDA), problems with CDC testing kits for states $[14,15]$, and early strict criteria regarding symptoms and exposure required to be tested. Some health systems had testing restrictions (e.g., requiring a physician on record to share test results, requiring government-issued ID, or only allowing drive-through tests) that disproportionately impacted minority and low-income communities who are already less likely to have a usual source of care. Across the country, community leaders (many clinicians themselves) stepped up. For example, leaders advocated for testing sites in lower-resourced communities in San Diego [16-18] where many essential workers live. Others simply established their own mobile outreach [19] to reach high-risk populations without access to health care or cars for drive-through testing. By June 2020, there were still very limited retail locations [20, 21] offering COVID-19 testing (1300 out of 32,000 possible nationwide locations) and even in December 2020, a large-scale, national coordinated testing strategy with rapid results has yet to be fully realized.

Partnering with community leaders and organizations not only helps to increase access to testing but also helps to address long-standing issues with mistrust and mistreatment in health care. Studies have shown that Blacks are less likely than Whites to trust their physicians [22] and are more likely to harbor trust in less formal sources of health information [23]. These differences are driven by a confluence of reasons including historic and personal experiences with mistreatment in clinical setting and unethical research. One large health care system found that unlike other racial/ethnic groups, a larger proportion of African Americans were tested for COVID-19 at the hospital [24] (inpatient or emergency department) rather than in an ambulatory 
setting, and they also were more likely to have symptoms severe enough to be hospitalized, indicating a possible delay in obtaining care. Another study found relatively high case fatality rates for Asian Americans [25] that may indicate underscreening and undertreatment due to ongoing discrimination related to origins of the virus.

Community organizations have also taken the lead in addressing related disparities in the workplace, especially for essential workers who lack sufficient paid sick leave to quarantine while waiting for test results or lack sufficient personal protective equipment (PPE) to ensure safety in the workplace. Spanish-speaking essential workers have faced barriers to obtaining PPE or even speaking to state investigators [26] about a COVID-19 outbreak at work. These issues not only exacerbate disparities within vulnerable populations but they also increase the exposure risks for the general community.

The disparities in the risk of infection extend from patients to health care providers. A prospective study [12] found that compared to the general population, minority health care workers have a greater risk of COVID-19 infection, even greater than non-Hispanic/Latinx White health care workers (adjusted hazard ratio 1.81, CI 1.45-2.24). Unsurprisingly, this study also found that minority health care workers were more likely than non-Hispanic White health care workers to report insufficient or reuse of PPE $(36.7 \%$ vs. $27.7 \%)$. Minority health care workers were also more likely to work in higher-risk settings (such as nursing homes) and be more likely to report caring for patients with coronavirus infection [12].

\section{New Research Initiatives and Resources to Address Health Disparities}

\section{National COVID Cohort Collaborative (N3C) and Disparities}

The recently established $\mathrm{N} 3 \mathrm{C}$ represents a multi-institutional collaborative initiative to develop a robust database of COVID-19 information drawn from EMRs. The goal of the consortium is to leverage such critical data elements to inform clinical decision-making, clinical trial design and implementation, as well as translational science investigation. The N3C Consortium implemented an inclusive phenotype standard for suspected and verified COVID-19 cases based on CDC guidelines [27]. The data are available in a secure enclave equipped with analytical tools where researchers can access and analyze the data but they cannot download. In reviewing de-identified data in the $\mathrm{N} 3 \mathrm{C}$ enclave (February 19, 2021), we found that the dataset contained demographics and clinical data for 3,082,743 patients from 41 contributing institutions. The analysis covered the demographic distribution of patients' data who were tested for SARS (severe acute respiratory syndrome)-CoV-2 (1,595,011 patients), diagnosed with the disease $(715,124$ patients), as well as hospitalized (115,330 patients), and died from the disease (60,970 patients not all deaths are verifiably caused by COVID-19). Approximately 55\% percent of the patient population was female. A similar gender ratio was found among the tested, COVID-19positive and hospitalized patients, while more male patients died of the disease (55\%) than female (45\%). The rate of positive tests was higher among Blacks (19\%) as compared to Whites (16.3). Hospitalization and mortality rates were also higher among Blacks. Hispanic/Latinx patients made up about $9 \%$ of the patient population and had significantly higher positivity and hospitalization rates than non-Hispanic/Latinx, while their
Table 1. Distribution of COVID-19 test positivity, hospitalization, and mortality (based on N3C Database, February 19, 2021)

\begin{tabular}{lccc}
\hline & $\begin{array}{c}\text { Test positivity } \\
\text { rate }\end{array}$ & $\begin{array}{c}\text { Hospitalization } \\
\text { rate }\end{array}$ & $\begin{array}{c}\text { Mortality } \\
\text { rate }\end{array}$ \\
\hline Female & $19.16 \%$ & $33.08 \%$ & $19.11 \%$ \\
\hline Male & $18.19 \%$ & $30.85 \%$ & $13.41 \%$ \\
\hline White & $16.30 \%$ & $28.28 \%$ & $19.13 \%$ \\
\hline African American & $19.01 \%$ & $34.67 \%$ & $19.58 \%$ \\
\hline $\begin{array}{l}\text { Non-Hispanic African } \\
\text { American }\end{array}$ & $18.59 \%$ & $35.91 \%$ & $20.53 \%$ \\
\hline $\begin{array}{l}\text { Non-Hispanic African } \\
\text { American male }\end{array}$ & $18.73 \%$ & $37.70 \%$ & $25.31 \%$ \\
\hline $\begin{array}{l}\text { Hispanic or Latino } \\
\text { Hinc }\end{array}$ & $30.59 \%$ & $43.13 \%$ & $6.25 \%$ \\
\hline
\end{tabular}

death rate was lower (Table 1). The patient population's age distribution had a median of 43 years. The median age of hospitalized patients was 48 years, while the median death age was 70 years. About $3 \%$ of the patients were diagnosed with COVID-19 without testing, and this was more prevalent in the early months of the pandemic when testing was less available. The ratio of diagnoses without testing was higher among non-Hispanic Blacks than among Whites. Compounding effects of demographic features were also analyzed to identify disparities affecting subcategories of populations, showing that the most vulnerable population was non-Hispanic Black males with 38\% hospitalization rate and $25 \%$ mortality.

While the $\mathrm{N} 3 \mathrm{C}$ database is an unprecedented resource with great potential, it currently has some limitations regarding disparities research. Participating institutions are mainly academic medical centers; therefore, the database lacks information from smaller community health centers. Data are consolidated from 41 different EMR systems (another 40 institutions are currently in the process of joining), which creates major challenges regarding data consolidation and quality. For example, $13 \%$ of the patients in the $\mathrm{N} 3 \mathrm{C}$ database do not have information on patient race or ethnicity. $\mathrm{N} 3 \mathrm{C}$ is working with participating institutions to improve data quality.

A number of domain teams have been created to researchspecific aspects of the pandemic, including elder impact and social determinants of health. The research developed by these domain teams using N3C data may be able to address some basic questions regarding health disparities for COVID-19. (https://covid.cd2h. org/domain-teams). The active social determinants of health domain team is working on linking publically available datasets to patient-level data.

As we continue to navigate this pandemic, it is important to note the importance of data collection analyses, and reporting on race/ethnicity especially given the early challenges with reporting infection, morbiditiy and mortality by race and ethnicity in the early months of the pandemic. First, a standardized national approach to data collection on race/ethnicity [28] must be sufficiently disaggregated to reveal important disparities that need to be addressed in both clinical care and research. For example, many states and counties still do not analyze COVID-19 data for Native Hawaiians and Pacific Islanders, but those that do [29] have found case rates that are among the highest or near highest across all racial/ethnic groups. In addition, a recent news article pointed out that $4 \%$ of the country's nursing staff are Filipino [30], yet they make up one-third of all COVID-19 deaths among nurses; this 
disparity would be otherwise overlooked since Filipinos are often grouped as "Asian" or "Other." Recently, the American Heart Association published some standard recommendations regarding the role of scientific journals in reporting racial and ethnic disparities and best practices for publishing disparities research [31].

Second, any data on COVID-19 disparities must be presented with contextual factors to avoid blaming individual patients for structural or social factors outside of their control or to avoid biologic explanatory models of health inequities. As the pandemic continues to reveal disparities, it also perpetuates a long unfortunate history of stereotypes of immigrants and racial/ethnic minorities as unclean or germ-ridden [4] rather than identifying the root cause of structural racism. Constant messaging about handwashing to prevent COVID-19 can also unintentionally imply that those who test positive for the virus do not follow proper hygiene [32].

\section{Demonstrating the Trustworthiness of the Research Enterprise}

As demonstrated by N3C, the COVID-19 pandemic has led to unprecedented levels of scientific cooperation and communication, as well as heightened public awareness of, and involvement in biomedical research [33]. In response to the global crisis, researchers and health care providers have rapidly engaged in collaborative, interdisciplinary work and shared important findings through rapid discoveries and dissemination of findings [34]. This cooperation has resulted in remarkable progress of therapeutic and preventive innovations, the speed of which has far outpaced expectations. The level of public attention to research is also at an all-time high [35]. The pandemic, public health, health care, and research enterprise addressing the pandemic are front and center on every news program and media outlet. Never have so many epidemiologists, public health scientists, immunologists, and virologists been in the public eye, reporting on previously esoteric topics such as immune response, herd immunity, statistical modeling, and vaccine mechanisms. However, politically driven interests, misinformation, and unclear messaging have clouded the picture presented to the public, and therefore the scientific community has also had to cope with tremendous misinformation, mixed messages, and the resulting cynicism toward science and research in a time that some would call a "mis-infodemic" [36].

\section{Importance of Community Partnerships}

Almost in tandem, the scientific community, policy-makers, and the general public all recognized how COVID-19 is disproportionately affecting communities of color [6]. We are all bearing witness, in real time, to the tremendous social and health disparities that affect individuals who have limited access to resources and limited options for COVID-19 mitigation. Researchers and community advocates have a unique opportunity to shine a light on longstanding social and health inequities faced by historically marginalized communities, highlight underlying structural drivers of inequality, and for the public to experience empathy for essential workers and their families. As we all experience the seismic shifts in our personal and professional lives, it is clear some families do not have the option to work from home or to receive an unemployment check, children cannot home-school because they lack access to affordable broadband, families cannot protect each other because a worker must take public transportation, or homes are too small to separate exposed individuals [3]. We need to seize the moment and leverage the sensitizing of scientists, clinicians, policy-makers, and the public to make lasting changes that will re-orient the translational research enterprise and reduce inequities.

It is important, however, that we understand that lasting changes are only possible by rebuilding trust with communities and demonstrating the trustworthiness of the scientific community [37, 38]. Trust, however, does not lend itself to "warp-speed" rebuilding efforts, particularly in racial/ethnic minority populations [39]. In order to demonstrate trustworthiness, researchers and the academic institutions they represent must renew their commitment to cultivating and sustaining a culture of scientific transparency, ethical conduct, and responsible community engagement $[38,40]$. This requires that we engage community members early on, value their lived experience and knowledge of community norms, are transparent with our motives, share our data, and ultimately promote power balance [38, 39]. A community-engaged partnership highlights the shared values of the scientific community and the general public: public health, safety, and inclusivity [37]. Clinical and Translational Science Awards (CTSAs) have recognized the importance of community and stakeholder engagement in fostering the success of translational research and now emphasize these as essential programs in their structures.

The demonstrated challenges in enrolling individuals from historically marginalized communities in clinical trials for SARS-CoV2 vaccines and novel therapeutics and promoting acceptance and uptake of these innovations once approved highlight the duality of true engagement. Despite well-deserved mistrust and "vaccine hesitancy" in communities of color, community leaders report that individuals are hopeful as vaccines are developed [41]. Lessons from past vaccination efforts, such as the human papillomavirus (HPV) vaccine, demonstrate that community-based approaches will be essential to success in achieving higher levels of uptake in diverse populations for a vaccine fraught with controversy [42]. The same trend is seen in preventive care interventions for cervical [43], prostate [44], and colon cancers [45], and diabetes [46].

\section{CEAL, RADX-UP, and other NIH initiatives and interactions with CTSA}

The National Institutes of Health (NIH) recognized both the need and the opportunity to leverage existing academic-community research partnerships and to create nationwide coordinated networks of scientists to address COVID-19 disparities through the Community Engagement Alliance (CEAL) Against COVID-19 Disparities, and the Rapid Acceleration of Diagnostics in Underserved Populations (RADx-UP) Initiatives [47]. Such coordinated initiatives can serve as models for future advances in health disparities research and provide the infrastructure for true community engagement in future prevention and therapeutic research. CEAL, led by the National Institute on Minority Health and Health Disparities (NIMHD) and National Heart, Lung, and Blood Institute (NHLBI), aims to combat COVID-19 misinformation, to rebuild trust in the research enterprise, and to encourage clinical trial recruitment and prevention behaviors in minority communities [47]. Established health disparities scientists from 11 states that were COVID-19 disparities "hot spots" were invited to propose state-wide projects to (1) conduct outreach and seek input from communities to raise awareness about COVID-19 and to address misinformation and mistrust about the pandemic and efforts to combat it, and (2) work to ensure that COVID-19 prevention and treatment clinical trials include racial and ethnically 
diverse communities most affected by the pandemic. The CEAL initiative, funded in September 2020, is bringing scientists together to plan and quickly implement collaborative outreach efforts to help mitigate the impact of COVID-19 on vulnerable populations and to evaluate their efforts through community-engaged research [48]. RADx-UP, led by the NIMHD and NIA, was a limited competition opportunity open to NIH-funded center and program grants with established community engagement infrastructures to build the evidence base of approaches to identify and address health disparities in COVID-19 diagnostic testing uptake and effectiveness in underserved populations. RAD-x-UP has so far funded over 70 research projects across the country.

Both the CEAL and RADx-UP initiatives include coordinating centers that facilitate cross-project collaborative research through sharing of resources, use of common metrics, and convening initiative-wide workgroups. The challenge, however, remains in finding adequate, evidence-based strategies that are generalizable and usable across sites but are also customizable to meet the unique needs and characteristics of diverse communities. Although the RADx-UP and CEAL initiatives have been proposed by specific agencies, each initiative has to some extent engaged the resources and infrastructure of local CTSAs that have institutional resources in community engagement and recruitment programs, education and training initiatives, and others. Networked research initiatives can also play an important role in informing preparedness efforts that may improve responses to future public health emergencies. However, these networks require support from a coordinating entity, such as being provided by CEAL and RADx-UP. Given its successful establishment of national workgroups and strategic management of networks that facilitate effective, scientific exchange, the CTSA may be well positioned to lead or support coordination efforts and play a unique role in shaping policies and models of care informed by the findings of the CEAL and RADx-UP initiatives that will ultimately improve the health of diverse communities.

Other CTSA-related, trans-NIH initiatives have included major efforts to collect data across multiple populations that are inclusive of underrepresented groups. NHLBI has funded an effort to add COVID-19-specific standardized data collection efforts to ongoing longitudinal cohort studies, including those that have large samples of Hispanics and Blacks. These studies can provide important data on COVID-19 associations with comorbidities, social determinants of health, and long-term outcomes. CTSAs have been effectively engaged in multiple therapeutic COVID-19 trials involving convalescent plasma and other investigational agents. Utilizing various CTSA programs including community and stakeholder engagement, integrating special populations, and participant and clinical interactions components, these centers have enhanced the enrollment of underrepresented groups in these trials.

\section{New Care Models to Impact Policy and Address Disparities}

\section{Leveraging Telehealth}

The COVID-19 pandemic has driven an extraordinary growth of telehealth. At the onset of the pandemic, most of the USA had "stay at home" orders in place, intended to reduce nonessential travel and exposure to protect individuals and preserve health care capacity. Consequently, routine health care visits were restricted, with many systems converting to telehealth for outpatient care despite limited effectiveness data on its use [49]. Unfortunately, there was significant concern that this approach would be accompanied by increasing health disparities. The digital divide, identified as the absence of necessary hardware and software, limited digital literacy, and/or the lack of reliable Internet access, disproportionately impacts populations also known to have higher rates of health disparities [50]. Early results have shown that older Americans, rural, and minority populations received less telehealth during the pandemic [51]. Compared to other age groups, patients older than 65 years had the lowest odds of using telehealth, and by race and ethnicity, Black and Hispanic/Latinx patients had lower odds of using telehealth versus emergency departments or an office visit than Whites or Asians, controlling for comorbidities and preferred language [52]. Even more concerning is that these are the same populations at higher risk for worse outcomes due to COVID-19 [49]. A significant number of populations are considered underserved or historically marginalized due to known health disparities or barriers to health care access, as well as populations medically and/or socially vulnerable due to COVID-19. Although in theory telehealth could help address many of the challenges populations with health disparities face, lack of transportation, limited health care access and the digital divide complicate telehealth's ability to do so. Further, in a time when health care systems are challenged to expedite fledgling telehealth programs, an important research agenda would focus on demonstrating the health outcomes and long-term impact of telehealth in historically underserved populations.

Beyond telemedicine aimed at individual patients, the pandemic has given rise to greater focus on tele-educational opportunities to inform health care providers and administrators of the latest best practices in emergency preparedness and treatment of COVID-19 [53]. The COVID-19 pandemic has created an infodemic, defined as an overabundance of information. According to the World Health Organization, an infodemic "makes it hard for people to find trustworthy sources and reliable guidance when they need it... like pathogens in epidemics, misinformation spreads further and faster and adds complexity to health emergency response" [54]. One example of tele-education is Project $\mathrm{ECHO}$, a powerful model designed to synthesize accurate and high-quality information and disseminate medical knowledge that can help address the infodemic. Created at the University of New Mexico in 2004, the ECHO model utilizes expertise uniquely positioned in academic medical centers to train community clinics in providing guideline-concordant care [55]. Currently, 83 Project ECHO sites have deployed COVID-19-specific ECHO series, reaching 16,535 participants representing 6000 different organizations [55]. A translational research agenda would examine the impact of these programs and how best to adapt and scale up future programming to meet crisis needs.

\section{Future Partnerships with CHWs}

CHWs are uniquely poised to help address health disparities created or exacerbated by the pandemic, as well as engage racial and ethnic minorities in research. CHWs have been demonstrated to improve health outcomes, particularly for underserved populations, in a variety of community-based interventions [56-58]. Because of their intimate connections with the community, CHWs can also serve as liaisons between investigators and historically marginalized populations in research throughout the translational spectrum from clinical to population health and policy research, especially for emerging topics like COVID-19. Further, 
CHWs can engage individuals from historically marginalized communities, understand and provide expertise in the context in which health problems exist, and serve as advisors on and advocates for their communities' needs [56]. CHWs can also address critical barriers to participation of underrepresented populations to participate in research by addressing fear and mistrust [58-60]. However, understanding the impact of CHWs in addressing COVID-19 disparities [61] is an area ripe for research with clear practice and policy implication and application of results. They are well positioned to help achieve CDC priorities of addressing disparities by countering misinformation and stigma related to COVID-19 in underserved communities. During the COVID-19 pandemic, CHWs have had to transform the way they connect with communities to adhere to stay-at-home orders and social distancing guidelines as recommended by the CDC [62]. For example, the work of many CHWs who are accustomed to having a physical presence in the community has been suddenly halted [63]. As new models of care are developed post-pandemic, the study of CHWs can help provide important findings for integration of care models to reduce disparities. Further, $\mathrm{CHWs}$ have the opportunity to help advance research initiatives to better understand and address COVID-19's unequal effects. As part of a diverse responder workforce, $\mathrm{CHW}$ s may serve as an important bridge between communities with health disparities and investigators focused on translational research in these populations. However, there remain several barriers preventing CHWs from engaging in research, including lack of research training in $\mathrm{CHW}$ certification programs and challenges associated with CHWs shifting to virtual work due to the COVID-19 pandemic. Overcoming these challenges requires the development and implementation of innovative research and remote work training, embedded in $\mathrm{CHW}$ training programs and as continuing education to previously trained $\mathrm{CHWs}$.

\section{Leveraging Geospatial Information Systems for Vaccine Distribution}

In the past, health geography has been used to measure geographic accessibility to health-related services like primary health centers and family planning services to promote equitable distribution of resources. Since the start of the pandemic, researchers have leveraged the spatial qualities of COVID-19 to model the spread, containment, and evolution of the virus in communities. However, as vaccines become available, geospatial information systems may be of considerable use to state and local health departments by informing the strategic distribution plans of the vaccine, particularly among individuals belonging to higher risk and/or hard-to-reach vulnerable populations. These systems can also be used to identify gaps in vaccination coverage, promote vaccination capacity building, and serve as an evaluation tool in health systems. Spatial patterns of vaccination have also been successfully used to inform public policy that has led to increased flu, measles, and $\mathrm{HPV}$ vaccination rates. Leveraging the capacity of spatial software may be crucial to boosting vaccination campaigns that will contribute to population-level immunity.

\section{Financial Support to Academic Medical Centers and Public Health Facilities}

Many academic medical centers are actively engaged with safety net hospitals in helping to deliver care to underserved populations. Such health care facilities were strained during the COVID-19 surges and have had to prioritize acute and chronic care for these diverse communities, as well as work with federal and state suppliers for vaccine distribution to the populations they serve. Partnering with faith-based and other local organizations will be necessary to reach communities at greatest need. Moreover, Federally Qualified Health Centers, State Department of Health's community clinics, and other state-based vaccine distribution centers have been rapidly constituted or expanded to meet increased demands. All of these expanded activities to increase access to programs for COVID-19 care and prevention will require increased financial support to meet these growing demands and address health equity. Temporary policy to support the financing of such care during a pandemic crisis should become important models for long-term options to help reduce health disparities.

\section{Conclusion: A Path Forward}

As the USA continues to address COVID-19 including newly identified variants, coordination and collaboration across sectors, communities, federal, and nonfederal agencies and academic institutions remain critical. Partnerships between communities, federal agencies, and academic institutions will be of particular importance to the dissemination of research-based information and education, and access to both diagnostic testing and novel therapies. Programs such as N3C, CEAL, and RADx-UP may serve as long-term models for addressing health disparities across the spectrum of disease beyond COVID-19. Access to data from EMRs should be leveraged for the purposes of improved tracking of disparities in disease and care delivery as well as informing strategic priorities in community partnerships, implementation science, and research agendas. With continued advances in prevention and therapeutics, translation to advance health equity will rely on engagement with local experts and respected grassroot organizations. As we seek to address the challenges inherent in COVID-19 disparities, academic clinicians and researchers must continue to partner with and listen to community leaders and members who are most familiar with what communities actually need and academic institutions should invest additional resources into community outreach and engagement. Their voices have gone unheard for so long and this has helped to perpetuate growing injustices. Any conversations about potential mitigation strategies moving forward, whether related to testing, contact tracing, clinical trial participation, or vaccine uptake will need to include their concerns, questions, and other feedback in order to be successful. Activities that are community-driven will be more likely to succeed because they will be more likely to actually meet what is needed. We must learn from experiences early in the pandemic to avoid repeating these mistakes and work towards the mitigation of all disparities.

Acknowledgments. The project [publication] described was supported by CTSA award No. UL1TR002649 (VCU) from the National Center for Advancing Translational Sciences. Its contents are solely the responsibility of the authors and do not necessarily represent official views of the National Center for Advancing Translational Sciences or the National Institutes of Health.

This work was funded in part by the University of Rochester Center for Leading Innovation and Collaboration (CLIC), under Grant U24TR002260. CLIC is the coordinating center for the Clinical and Translational Science Awards (CTSA) Program, funded by the National Center for Advancing Translational Sciences (NCATS) at the National Institutes of Health (NIH). This work is solely the responsibility of the authors and does not necessarily represent the official views of the NIH. 
The project described was supported by the National Center for Advancing Translational Sciences, National Institutes of Health, through Grant UL1 TR002014. The content is solely the responsibility of the authors and does not necessarily represent the official views of the NIH.

Data analysis for this project was conducted with the support of the National COVID Cohort Collaborative (N3C)

Disclosures. The authors have no conflicts of interest to disclose.

\section{References}

1. Johns Hopkins Coronavirus Resource Center. COVID-19 United States cases by county. Johns Hopkins University \& Medicine, n.d. [cited Dec 30, 2020]. (https://coronavirus.jhu.edu/us-map)

2. Hatcher SM, Agnew-Brune C, Anderson M, et al. COVID-19 among American Indian and Alaska native persons - 23 States, Morbidity and Mortality Weekly Report 2020; 69(34): 1166-1169.

3. Nayak A, Islam S, Mehta A, et al. (2020). Impact of social vulnerability on COVID-19 incidence and outcomes in the United States. medRxiv 2020. doi: 10.1101/2020.04.10.20060962

4. Chowkwanyun M, Reed AL. Racial health disparities and COVID-19 caution and context. The New England Journal of Medicine 2020; 383: 201-203.

5. Rogers TN, Rogers CR, VanSant-Webb E, et al. Racial disparities in COVID-19 mortality among essential workers in the United States. World Medical \& Health Policy 2020. doi: 10.1002/wmh3.358

6. Webb Hooper M, Nápoles AM, Pérez-Stable EJ. COVID-19 and racial/ ethnic disparities. Journal of the American Medical Association 2020; 323(24): 2466-2467. doi: 10.1001/jama.2020.8598

7. Laurencin CT, Mcclinton A. The COVID-19 pandemic: a call to action to identify and address racial and ethnic disparities. Journal of Racial and Ethnic Health Disparities 2020; 7: 398-402. doi: 10.1007/s40615-02000756-0

8. Arasteh K. Prevalence of comorbidities and risks associated with COVID19 among black and Hispanic populations in New York City: an examination of the 2018 New York City community health survey. Journal of Racial and Ethnic Health Disparities 2020: 1-7. doi: 10.1007/s40615-020-00844-1

9. Richardson S, Hirsch JS, Narasimhan M, et al. Presenting characteristics, comorbidities, and outcomes among 5700 patients hospitalized with COVID-19 in the New York City area. Journal of the American Medical Association 2020; 323: 2052. doi: 10.1001/jama.2020.6775

10. Community Health Workers. apha.org [cited Oct 19, 2020]. (https://www. apha.org/apha-communities/member-sections/community-health-workers)

11. Santarone K, McKenney M, Elkbuli A. Preserving mental health and resilience in frontline healthcare workers during COVID-19. American Journal of Emergency Medicine 2020; 38(7): 1530-1531. doi: 10.1016/j.ajem.2020. 04.030

12. Nguyen LH, Drew DA, Graham MS, et al. Coronavirus pandemic epidemiology consortium. risk of COVID-19 among front-line health-care workers and the general community: a prospective cohort study. Lancet Public Health 2020; 5(9): e475-e483. doi: 10.1016/S2468-2667(20)30164-X

13. Mayfield-Johnson S, Smith DO, Crosby SA, et al. Insights on COVID-19 from community health worker state leaders. The Journal of Ambulatory Care Management 2020; 43(4): 268-277. doi: 10.1097/JAC.000000000 0000351

14. Dudzinski DM, Yoisington BY, Brown CE. Ethics lessons from Seattle's early experience With COVID-19. The American Journal of Bioethics 2020; 20(7): 67-74.

15. Shear MD, Goodnough A, Kaplan S, et al. The Lost Month: How a Failure to Test Blinded the U.S. to COVID-19. The New York Times, March 28, 2020, updated April 1, 2020.

16. Lopez-Villafaña A. Advocates call on county to open frequent COVID-19 testing sites in southeast San Diego. The San Diego Union Tribune, April 29, 2020.

17. Lopez-Villafaña A. COVID-19 cases higher among San Diego's Latinos; advocates call for more testing. Los Angeles Times, May 7, 2020.
18. Lopez-Villafaña A. Community advocates applaud opening of COVID-19 testing site in southeast San Diego. The San Diego Union Tribune, May 20, 2020.

19. Attipoe-Dorcoo S, Delgado R, Gupta A, et al. Mobile health clinic model in the COVID-19 pandemic: lessons learned and opportunities for policy changes and innovation. International Journal for Equity in Health 2020; 19(1): 73. doi: 10.1186/s12939-020-01175-7

20. Feldman N. Black Doctors Work to Make Coronavirus Testing More Equitable. Kaiser Health News, October 13, 2020.

21. Pfeiffer S, Mak T. Trump's Plan for Drive-Up COVID-19 Tests At Stores Yields Few Results. NPR Morning Edition, June 1, 2020.

22. Boulware LE, Cooper LA, Ratner LE, et al. Race and trust in the health care system. Public Health Reports 2003; 118(4): 358-365.

23. Musa D, Schulz R, Harris R, et al. Trust in the health care system and the use of preventive health services by older black and white adults. American Journal of Public Health 2009; 99: 1293-1299.

24. Azar KMJ, Romanelli RJ, Lockhart SH, et al. Disparities in outcomes among COVID-19 patients in a large health care system in California. Health Affairs 2020; 39(7): 1253-1262. doi: 10.1377/hlthaff.2020.00598. Epub May 2, 202.

25. Yan BW, Ng F, Chu J, Tsoh J, Nguyen T. Asian Americans facing high COVID-19 case fatality. Health Affairs Blog, July 13, 2020.

26. Hussein F, Douglas G. Language Barriers Pose Worker Rights Pitfalls During Pandemic. Bloomberg Law: Occupational Safety \& Health Reporter, October 9, 2020.

27. Haendel M, Chute C, Gersing K. The National COVID Cohort Collaborative (N3C): rationale, design, infrastructure, and deployment. Journal of the American Medical Informatics Association 2020: ocaa196. doi: 10.1093/jamia/ocaa196

28. Williams V. U.S. slow to collect complete data on race, ethnicity in coronavirus cases, civil rights group says. The Washington Post, May 22, 2020.

29. Chang RC, Penaia C, Thomas K. Count Native Hawaiian and Pacific Islanders in COVID-19 Data - It's an OMB Mandate. Health Affairs Blog, August 27, 2020.

30. Akhtar A. Filipinos make up $4 \%$ of nurses in the US, but $31.5 \%$ of nurse deaths from COVID-19. Business Insider, September 29, 2020.

31. Breathett K, Spatz ES, Kramer DB, et al. The groundwater of racial and ethnic disparities research: a statement From Circulation: Cardiovascular Quality and Outcomes. Circulation: Cardiovascular Quality and Outcomes 2021; 14(2): e007868. doi: 10.1161/CIRCOUTCOMES.121.007868

32. Bischoff LA. Lawmaker asks if 'colored population' not washing their hands as well as others behind COVID rates. Dayton Daily News, June 23, 2020 .

33. Palamenghi L, Barello S, Boccia S, Graffigna G. Mistrust in biomedical research and vaccine hesitancy: the forefront challenge in the battle against COVID-19 in Italy. European Journal of Epidemiology 2020; 35(8): 785-788. doi: 10.1007/s10654-020-00675-8. Epub 2020 Aug 17

34. Lee J, Haupt J. (2020). Scientific globalism during a global crisis: research collaboration and open access publications on COVID-19. Higher Education. doi: 10.1007/s10734-020-00589-0

35. Evans J, Hargittai E. Who doesn't trust fauci? The public's belief in the expertise and shared values of scientists in the COVID-19 pandemic. SOCIUS (2020); 6: 1-13.

36. McGinty M, Gyenes $\mathbf{N}$. A dangerous misinfodemic spreads alongside the SARS-COV-2 pandemic. The Harvard Kennedy School (HKS) Misinformation Review 2020.

37. Wilkins C. Effective engagement requires trust and being trustworthy. Medical Care 2018; Oct; 56 Suppl 10 Suppl 1(10 Suppl 1):S6-S8 doi: 10. 1097/MLR.0000000000000953.

38. Chatfield K, Schroeder D. Ethical research in the COVID-19 era demands care, solidarity and trustworthiness. Research Ethics 2020; 16(3-4): 1-4.

39. Sprague-Martinez L, Carolan K, O'Donnell A, Diaz Y, Freeman E. Community engagement in patient-centered outcomes research: Benefits, barriers and measurement. Journal of Clinical and Translational Science 2018; 2(6): 371-376.

40. Brown S, Partee P, Fend J, et al. Outreach to diversify clinical trial participation: a randomized recruitment. Clinical Trials 2015; 12(3): 205-211. 
41. Harrison E, Wu J. Vaccine confidence in the time of COVID-19. European Journal of Epidemiology; 2020; 35: 235-330.

42. Carnegie E, Whittaker A, Brunton C, et al. Development of a cross cultural HPV community engagement model within Scotland. Health Education Journal 2017; 398-410.

43. Wood B, Burchell AN, Escott N, et al. Using community engagement to inform and implement a community-randomized control trial in the anishinaabek cervical cancer screening study. Frontiers in Oncology. 2014; 4: 27

44. Jackson D, Owens O, Friedman D, Dubose-Morris R. Innovative and community-guided evaluation and dissemination of a prostate cancer education program for African American men and women. Journal of Cancer Education 2015; 779-785.

45. Davis M, Freeman M, Shannon J, et al. A systematic review of clinic and community intervention to increase fecal testing for colorectal cancer in rural and low-income populations in the United States - How, what and when? BMC Cancer 2018; 18: 40. Published online Jan 6, 2018. doi: 10. 1186/s12885-017-3813-4.

46. Feathers J, Kieffer E, Palmisano G, et al. The development, implementation, and process evaluation of the REACH Detroit Partnership's Diabetes Lifestyle Intervention. Diabetes Education; May-Jun 2007; 33(3): 509-520. doi: $10.1177 / 0145721707301371$.

47. NIH. COVID-19 Community, September 2, 2020. Retrieved from The Community Engagement Alliance (CEAL) Against COVID-19 Disparities. (https://covid19community.nih.gov/about/)

48. NIH. NIH Funds Community Engagement Efforts in Areas Hardest Hit by COVID-19. Bethesda, MD: NIH, 2020, September 16.

49. Wosik JA, Fudim M, Cameron B, et al. Telehealth transformation: COVID-19 and the rise of virtual care. Journal of the American Medical Informatics Association 2020 Apr 20. doi: 10.1093/jamia/ocaa067.

49. Velasquez D, Mehrotra A. Ensuring The Growth Of Telehealth During COVID-19 Does Not Exacerbate Disparities In Care. Health Affairs Blog, May 8, 2020. doi: 10.1377/hblog20200505.591306

50. Miller SJ, Astha V, Janevic T, Benn E. Characteristics of telehealth users in NYC for COVID-related care during the coronavirus pandemic. Journal of the American Medical Informatics Association, 2020; 27(12): 1949-1954. doi: 10.1093/jamia/ocaa216

51. Aldridge RW, Lewer D, Katikireddi SV, et al. Black, Asian and Minority Ethnic groups in England are at increased risk of death from COVID-19: indirect standardisation of NHS mortality data. Wellcome Open Research 2020; 5(88): 88.
52. Pollock K, Setzen M, Svider PF. Embracing telemedicine into your otolaryngology practice amid the COVID-19 crisis: an invited commentary. American Journal of Otolaryngology 2020; 41(3): 102490. doi: 10.1016/j. amjoto.2020.102490

53. Humphreys J, Schoenherr L, Elia G, et al. Rapid implementation of inpatient telepalliative medicine consultations during COVID-19 pandemic. Journal of Pain and Symptom Management 2020; 60(1): e54-e59. doi: 10.1016/j.jpainsymman.2020.04.001

54. E. World Health Organization. 1st WHO Infodemiology Conference. 30 June - 16 July 2020 [cited Aug 13, 2020]. (https://www.who.int/newsroom/events/detail/2020/06/30/default-calendar/1st-who-infodemiologyconference)

55. Arora S, Thornton K, Komaromy M, Kalishman S, Katzman J, Duhigg D. Demonopolizing medical knowledge. Academic Medicine 2014; 89(1): 30-32. doi: 10.1097/ACM.0000000000000051

56. Andrews JO, Felton G, Wewers ME, Heath J. Use of community health workers in research withethnic minority women. Journal of Nursing Scholarship 2004; 36(4): 358-365.

57. Rhodes SD, Foley KL, Zometa CS, Bloom FR. Lay health advisor interventions among Hispanics/Latinos: a qualitative systematic review. American Journal of Preventive Medicine 2007; 33(5): 418-427.

58. Viswanathan M, Kraschnewski JL, Nishikawa B, et al. Outcomes of community health worker interventions. Evidence Report/Technology Assessment 2009; 181: 1-144, A1-A2, B1-B14, passim.

59. Ulrich A, Thompson B, Livaudais JC, Espinoza N, Cordova A, Coronado GD. Issues in biomedical research: what do Hispanics think? American Journal of Health Behavior 2013; 37(1): 80-85.

60. Schmotzer GL. Barriers and facilitators to participation of minorities in clinical trials. Ethnicity \& Disease 2012; 22(2): 226-230.

61. Corbie-Smith G, Thomas SB, Williams MV, Moody-Ayers S. Attitudes and beliefs of African Americans toward participation in medical research. Journal of General Internal Medicine 1999; 14(9): 537-546.

62. Smith DO, Wennerstrom A. To strengthen the public health response to COVID-19, we need community health workers. Health Affairs Blog, May 6, 2020 [cited Oct 19, 2020]. (https://www.healthaffairs.org/do/10.1377/ hblog20200504.336184/full/)

63. Peretz PJ, Islam N, Matiz LA. Community health workers and Covid-19 addressing social determinants of health in times of crisis and beyond. The New England Journal of Medicine 2020; 383(19): e108. doi: 10.1056/ NEJMp2022641 